\title{
Tracking Internal Temperature and Structural Dynamics during Nail Penetration of Lithium-Ion Cells
}

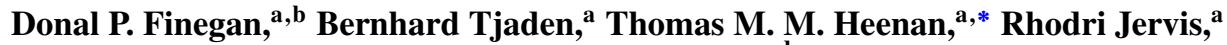 \\ Marco Di Michiel, ${ }^{\mathrm{c}}$ Alexander Rack, ${ }^{\mathrm{c}}$ Gareth Hinds, ${ }^{\mathrm{d}}$ Dan J. L. Brett, ${ }^{\mathrm{a}}$ and Paul R. Shearing ${ }^{\mathrm{a}, \mathrm{z}}$
}

${ }^{a}$ Electrochemical Innovation Lab, Department of Chemical Engineering, University College London, London WC1E

7JE, United Kingdom

${ }^{b}$ Transportation and Hydrogen Systems Center, National Renewable Energy Laboratory, Golden, Colorado 80401, USA

${ }^{c}$ ESRF The European Synchrotron, 38000 Grenoble, France

${ }^{d}$ National Physical Laboratory, Teddington, Middlesex TW11 OLW, United Kingdom

\begin{abstract}
Mechanical abuse of lithium-ion batteries is widely used during testing to induce thermal runaway, characterize associated risks, and expose cell and module vulnerabilities. However, the repeatability of puncture or 'nail penetration' tests is a key issue as there is often a high degree of variability in the resulting thermal runaway process. In this work, the failure mechanisms of 18650 cells punctured at different locations and orientations are characterized with respect to their internal structural degradation, and both their internal and surface temperature, all of which are monitored in real time. The initiation and propagation of thermal runaway is visualized via high-speed synchrotron X-ray radiography at 2000 frames per second, and the surface and internal temperatures are recorded via infrared imaging and a thermocouple embedded in the tip of the penetrating nail, respectively. The influence of the nail, as well as how and where it penetrates the cell, on the initiation and propagation of thermal runaway is described and the suitability of this test method for representing in-field failures is discussed.

(C) The Author(s) 2017. Published by ECS. This is an open access article distributed under the terms of the Creative Commons Attribution 4.0 License (CC BY, http://creativecommons.org/licenses/by/4.0/), which permits unrestricted reuse of the work in any medium, provided the original work is properly cited. [DOI: 10.1149/2.1501713jes] All rights reserved.

(cc) BY
\end{abstract}

Manuscript submitted September 5, 2017; revised manuscript received October 12, 2017. Published October 31, 2017.

Abuse tests of Li-ion batteries are applied to determine the risks associated with failure of a particular cell design. Examples of risks that are of interest to understand include: quantity and rate of heat and gas generation, maximum temperatures reached, how the generated heat is dissipated, and whether projectiles are produced. Carrying out an abuse test that is fully representative of in-field failures is challenging. Mechanical abuse is the most common method used to simulate in-field failures and is of particular interest to the automotive sector where crash and impact tests compromise the safety of utilized cells. Of these, the most common technique used to induce failure within cells and battery packs is via penetration of the cell with a sharp object such as a nail during so-called 'nail penetration' tests. When the positive and negative electrodes of a cell are connected by a material with low electrical resistance, the cell rapidly discharges with a high rate of Joule and entropic heat generation. When a critical temperature is exceeded locally, and if there is a sufficient amount of electrolyte, a series of exothermic reactions initiate and propagate throughout the cell in a process known as 'thermal runaway'. ${ }^{1-6}$

In-field puncture-induced failures incur a high degree of variability, which arises from difficulties in controlling parameters such as the internal architecture of the cell, the size, shape, electrical and thermal properties of the puncturing object, as well as the depth and rate at which the cell is punctured. When attempting to replicate a scenario in which a cell undergoes an internal short circuit, the nail penetration test is unsuitable due to it being inherently intrusive, spreading the short circuit across a large area and multiple layers, and introducing a heat sink at the region of initiation. For these reasons, the nail penetration test is not considered to be a suitable technique for reproducibly inducing in-field failures or worst case scenarios. Inducing an ondemand local failure within the cell that is representative of an infield failure poses a great challenge. ${ }^{7}$ Mechanical abuse using blunt objects can also lead to internal short-circuiting. For example, when mechanical failure of the electrode assembly occurs due to applied tensile strain, electrically conducting layers from the positive and negative electrode can make contact, causing an internal short circuit. ${ }^{8}$ Alternative methods for inducing internal short circuits by implanting devices during manufacturing have previously been described, ${ }^{8,9}$ but have not yet been accepted as standard test methods.

\footnotetext{
*Electrochemical Society Student Member
}

${ }^{\mathrm{z} E}$-mail: p.shearing@ucl.ac.uk
Numerous thermal, electrochemical and mechanical models have been developed to predict the response of internal architectures of commercial cells to mechanical deformation, ${ }^{10-13}$ but to date these have not been validated through direct observation of internal deformations during mechanically-induced thermal runaway. Maleki and Howard ${ }^{14}$ reported that a short circuit near the edge of the electrode assembly results in a greater temperature rise locally due to the heat being dissipated primarily in one direction, a study which was supported by surface temperature measurements and post-mortem inspection of the cell. Empirical results that elucidate the internal structural and thermal behavior are required to connect internal phenomena with external measurements and to validate multi-physics models. ${ }^{11,12,15,16}$ To understand the variation of failure mechanisms in response to different abuse conditions, a spatio-temporal description of thermal runaway resulting from each scenario is needed. High-speed X-ray computed tomography (CT) and radiography have been used in previous studies to visualize the internal breakdown and ejection of active materials following thermal runaway induced via thermal abuse, ${ }^{17}$ electrical overcharge $^{18}$ and internal short-circuiting. ${ }^{8}$ Failures induced via intrusive mechanical methods are expected to deviate most significantly from other in-field failures, in particular internal short-circuiting, which nail penetration is sometimes used to simulate.

In this work, visualization via high-speed X-ray imaging is used to describe the variation of 18650 cell behavior during nail penetration tests, providing insight into mechanisms of internal structural breakdown of cells. The propagation of thermal runaway from the point of initiation is captured using high-speed X-ray imaging at 2000 frames per second (fps), and a combined thermal mapping approach of infrared thermal imaging and internal temperature measurement, via a modified nail with an embedded thermocouple at its tip, ${ }^{19}$ is used to describe the thermal response of the cells. The variability of the thermal and internal structural behavior during nail penetration tests at different locations and orientations of 18650 cells is elucidated.

\section{Experimental}

Smart-nail design.-A custom made stainless steel 314 nail with an integrated rapid response insulated K-type thermocouple, that had a diameter of $0.5 \mathrm{~mm}$ and an operating range from $-100^{\circ} \mathrm{C}$ to $800^{\circ} \mathrm{C}$ (Product 406-534, TC-direct, UK) (Figure 1a), was used to measure the temperature at the tip of the nail during the tests. This design was inspired by the "smart-nail" described by Hatchard et al. ${ }^{19}$ but 


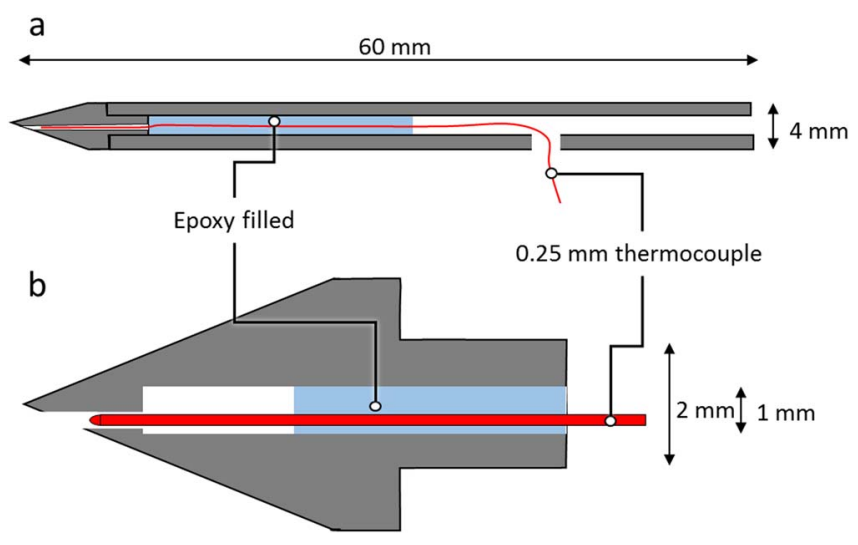

Figure 1. (a) Illustration showing a sectioned view of the stainless steel nail with an integrated thermocouple at its tip. The nail consisted of two components, the shaft and the tip. (b) Magnified view of the tip section showing the placement of the thermocouple just beneath the surface of the nail at a slightly offset position from the center of the sharpened tip. The tip section was forced into the shaft during assembly.

with some modifications. A $60 \mathrm{~mm}$ long stainless steel tube with an external diameter of $4 \mathrm{~mm}$ and an internal diameter of $2 \mathrm{~mm}$ was used as the shaft of the nail. A stainless steel tip (Figure 1b) was sharpened and shaped on a lathe. A $1 \mathrm{~mm}$ diameter bore was drilled in the tip to just beneath the front surface; thereafter a $0.5 \mathrm{~mm}$ diameter hole, which was slightly offset from the center of the tip, was drilled through to the surface. This insured that the tip of the nail remained sharp and that the punctured material would mostly glide over the off-centered hole as the nail continued to push through the cell. The complete tip component was forced into the cylindrical shaft. The $0.5 \mathrm{~mm}$ diameter thermocouple was coated in epoxy and fed through the assembly until it reached a subsurface location near the tip as shown in Figure 1. The subsurface placement of the thermocouple protected it from damage while also allowing it to have direct contact with the hot fluidized material within the cell during the penetration tests. This ensured representative temperature readings with a rapid response time. To avoid inaccurate temperature measurements arising from thermal or mechanical damage to the nail or thermocouple, a new nail assembly was used for each test.

Experimental set-up.-The nail penetration tests were carried out inside a commercial nail penetration system (MTI Nail Penetration Tester, MSK-800-TE9002, MTI, Richmond, CA, USA), that was modified to have X-ray transparent $2 \mathrm{~mm}$ thick aluminum front and rear panels for X-ray imaging, and an infrared-transparent $2 \mathrm{~mm}$ thick sapphire window for thermal imaging. Photographs of the setup in beamline ID19 at The European Synchrotron (ESRF) are provided as Supplementary Information. The nail penetration system was mounted on a heavy-duty sample stage that allowed position-adjustment in the vertical direction, and the containment was connected to an extraction fan to remove any smoke or harmful gases that were generated during the tests.

The 18650 lithium-ion cells (LG ICR18650S3) were held in place by hydraulic clamps that operated at 4 bar. The hydraulic piston nail penetrator was connected to a 5 bar air supply. All tests were carried out using the smart nail described above. The surface temperature of the 18650 cells was recorded using a thermal camera (FLIR SC5000MB, FLIR Systems France, Croissy Beaubourg, France). The thermal camera was operated in its high-temperature setting, which had a calibrated temperature range of $250^{\circ} \mathrm{C}-1500^{\circ} \mathrm{C}$. The thermal camera had an infrared detection range of $2.5 \mu \mathrm{m}$ to $5.1 \mu \mathrm{m}$ on an InSb detector, and was shielded against high-energy X-rays by sheets of lead. The thermal camera was positioned ca. $30 \mathrm{~cm}$ from the penetration location, on the outer side of the sapphire window. The sapphire window had a transmittance of ca. $86 \%$ in the infrared range of $2.5 \mu \mathrm{m}$ to $5.1 \mu \mathrm{m}$ (data presented as Supplementary Information). The 18650 cells were painted with a heat-resistant black paint with a calibrated emissivity of 0.96 . The nail penetration system and thermal camera were operated from the user-control room, outside the beam-hutch.

X-ray imaging. - High-speed X-ray imaging of 18650 cells undergoing nail penetration was carried out at beamline ID19 at the ESRF. A polychromatic beam was used with a LuAG: $\mathrm{Ce}\left(\mathrm{Lu}_{3} \mathrm{Al}_{5} \mathrm{O}_{12}: \mathrm{Ce}\right)$ scintillator and a high-speed PCO.Dimax camera (PCO AG, Germany). Two different frame rates were used: $2000 \mathrm{fps}$ with an exposure time of $457 \mu$ s and a field of view (FOV) of $2016 \times 1232$ pixels with a pixel resolution of $10 \mu \mathrm{m}$, and $5310 \mathrm{fps}$ with an exposure time of $44 \mu \mathrm{s}$, and FOV of $1344 \times 668$ pixels with a pixel resolution of $20 \mu \mathrm{m}$. Flat-field correction and time stamps were applied to the radiographs and the resulting images were compiled into movies using MATLAB. The radiography movies are provided as supplementary material where Supplementary Movie 6 was imaged at $5310 \mathrm{fps}$ and the rest of the supplementary movies were imaged at $2000 \mathrm{fps}$.

\section{Results and Discussion}

Two 18650 cells underwent nail penetration in each of the four orientations shown in Figure 2: Position 1 involved a horizontal penetration midway along the cell (Supplementary Movies 1-2), Position 2 involved an off-centered vertical penetration through the base of the cell (Supplementary Movies 3-4), Position 3 involved horizontal penetration near the cell-header (Supplementary Movies 5-6), and Position 4 involved an off-centered vertical penetration through the cell-header (Supplementary Movies 7-8). In all cases, penetration was offset from the longitudinal axis of the cell to avoid penetration into the vacant core where short-circuiting would not occur. To simultaneously assess the thermal and structural dynamics, spatially and temporally, an approach that combined thermal imaging, highspeed X-ray imaging, and internal temperature measurement (via the smart-nail design) was implemented.

Thermal behavior.-In Figure 2a, the time evolution of both the temperature at the tip of the nail (measured by the internal thermocouple) and the surface temperature of the cell in the region surrounding the nail (measured using the thermal camera) are compared for each of the four penetration locations. The temperature of the surface of the cell in each location was determined by taking an average value from a surface region (squared section within the thermal images in Figure 2a). The region surrounding the nail showed the highest surface temperature due to short-circuiting and initiation of thermal runaway occurring locally. The temperature within the cell varied significantly between the different test positions. The cells penetrated vertically (Position 2 and Position 4) exhibited the highest surface and internal temperatures where in both cases (penetration through the top and bottom) the maximum internal temperature was measured to be $>900^{\circ} \mathrm{C}$. Here, the surface temperature was around $300^{\circ} \mathrm{C}$ lower than the internal value. Such high internal temperatures during thermal runaway were previously inferred for similar cells ${ }^{17}$ by observing melting of copper current collectors (melting point of copper $1085^{\circ} \mathrm{C}$ ). The operating range of the thermocouple used here was up to $800^{\circ} \mathrm{C}$; above this value the measurements should be considered indicative only, albeit the recorded thermocouple response (Figure 2) did not show any sign of signal failure. Furthermore, the radiographs that are presented later in the text do not show any evidence of molten copper indicating that in these tests internal temperatures did not exceed $1085^{\circ} \mathrm{C}$. For Position 3 , the region at the tip of the nail cooled relatively quickly to approximately the surface temperature of the cell. This is explained by the nail deforming within the cell and coming to rest in the vacant region between the top of the electrode assembly and the cell-header. This can be clearly observed in the radiographs, which show the final position of the nail tip outside the reaction zone.

The temperature measured at the top, bottom and middle of the 18650 cells is highly dependent on the location of the nail penetration (Figure 2b). In three of the four positions (the exception being 

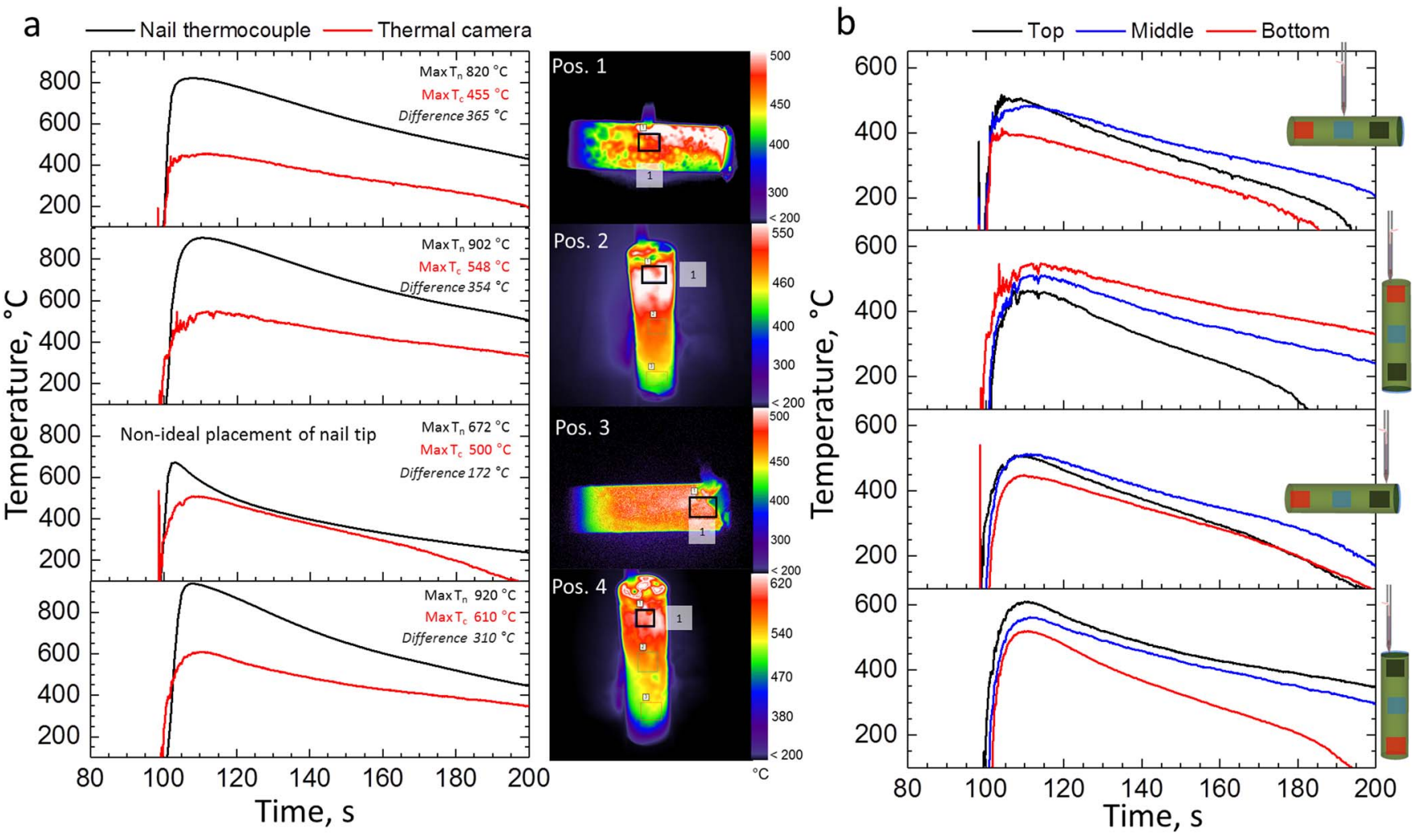

Figure 2. Nail penetration tests at four different locations on an 18650 cell: (a) Temperature profiles showing the difference between the temperature experienced by the 'smart nail' thermocouple $\left(T_{n}\right)$ and the surface temperature measured around the region of penetration by the thermal camera $\left(T_{c}\right)$. Thermal images showing the surface temperature profile and region in which the highest surface temperature was measured (labelled 1). (b) Surface temperature measured by the thermal camera at three longitudinal locations during penetration with inset illustrations showing the regions of measurement.

Position 2), the highest temperature was observed at the cell-header, whereas the lowest temperature was at the bottom of the cell. This demonstrates the importance of selective positioning of thermocouples to determine maximum surface temperatures reached during mechanical abuse tests.

For Position 1, the maximum temperature measured was found to be beneath the cell-header. Contrary to most thermal models of nail penetration, the heat dissipation within the cell is not isotropic. This anisotropic heat dissipation, and in particular, the maximum temperature achieved at the cell-header is rationalized in the following section by observing the internal structural dynamics using high-speed radiography. The molten degradation products transported the heat in the direction of the vent; the surface temperature profile observed in the thermal image for Position 1 is indicative of this, where the high temperature extends from the point of penetration toward the vent. Fluidization of reactants and materials is often not considered when modelling thermal runaway but plays an important role in propagation of thermal runaway and dissipation of heat. As seen in the anisotropic surface temperature profiles in Figure 1 and the internal structural dynamics during thermal runaway described in the following sections, it is imperative to include fluid dynamics in future multi-physics models of thermal runaway to accurately predict surface temperature profiles and local heat flux.

Internal structural damage.-Position 1. Initially, heat generation would only be expected to occur from Joule heating associated with short-circuiting at the interface between the nail and the electrodes. When a sufficiently high temperature was reached at the interface, the active materials began to decompose exothermically, leading to the propagation of thermal runaway from the interfacial area around which the short circuit was facilitated. This process is presented as timestamped radiographs in Figure 3 and in real-time in Supplementary Movie 1. In the raw radiographs (Supplementary Movies), the reaction zone appears as a locally reduced greyscale value and distortion of the electrode structure. This reaction zone is highlighted by a semitransparent red layer in the following figures. The initiation of thermal runaway appears to have occurred during indentation of the casing by the nail and prior to the nail puncturing the casing. Initially, a hard short is expected to have occurred where the cell discharged at the location where the nail punctured the casing. Thereafter, as the tip of the nail proceeded radially through the electrode layers, the effect of the short circuit was softened by the increasing surface area over which current could flow. This is observed by the relatively fast propagation of thermal runaway longitudinally along the outer layers of the electrode assembly between $1.9060 \mathrm{~s}$ and $2.1860 \mathrm{~s}$ in Figure 3.

At $1.9060 \mathrm{~s}$ in Figure 3, a crack is observed to have propagated from the inner layers of the electrode assembly toward the tip of the penetrating nail. The crack occurred in the azimuthal direction along the assembly, which is similar to what was modelled by Zhang. ${ }^{13}$ This may be due to the lower tensile strength of the separator material in the longitudinal direction relative to the azimuthal direction. ${ }^{20-22}$ Crack propagation across the layers in the radial direction is expected to have further increased the interfacial area between the positive and negative electrode facilitating the short circuit. With increased surface area over which the cell is short-circuiting, heat generation becomes more delocalized, reducing the peak temperature at hotspots. This is observed in Figure 2 where the surface temperatures are lowest for the positions involving penetration across numerous layers (Positions 1 and 3 ).

The vacant core of the cell allowed the electrode assembly to collapse inwards, which would increase the displacement required for puncture of subsequent layers, as suggested by Lamb and Orendorff. ${ }^{7}$ In Position 1 (Figure 3), the strain caused by displacement appears to be the cause of the tearing and shorting across the inner layers. From Supplementary Movie 1, the rate at which thermal runaway 


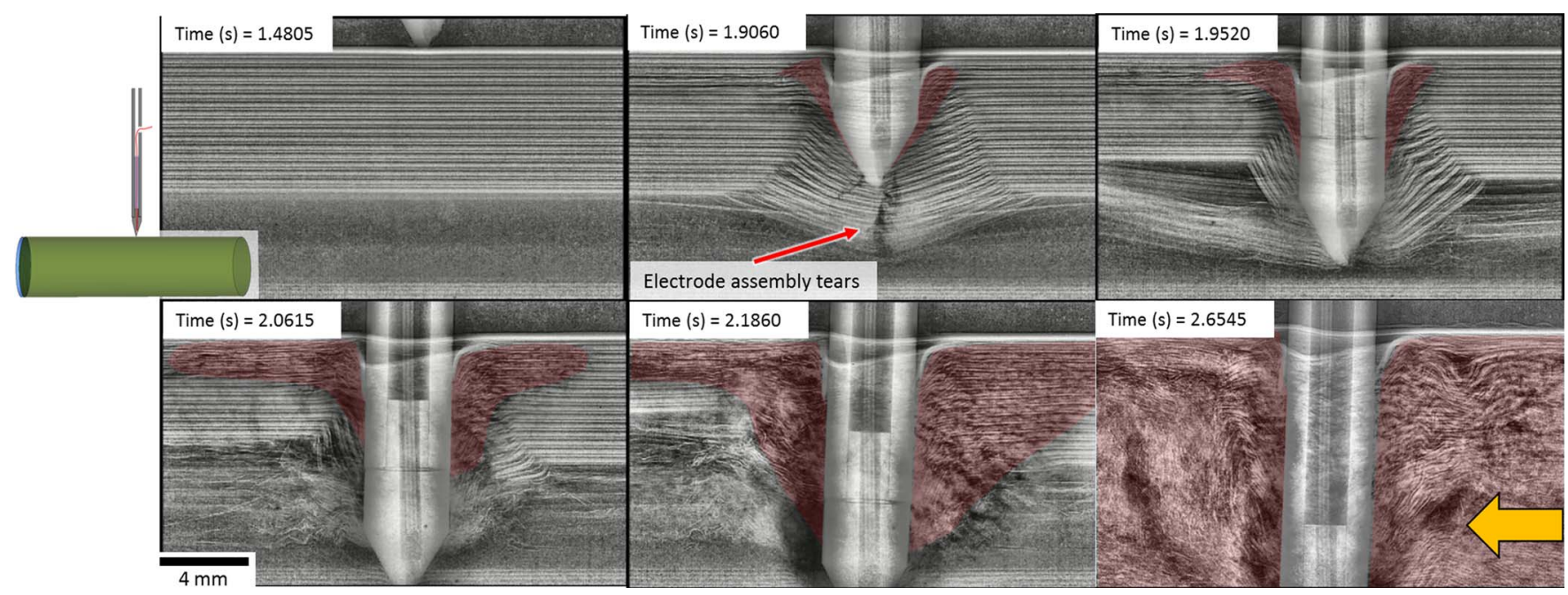

Figure 3. Time-stamped radiographs of nail penetration of an 18650 cell at Position 1 showing initiation and propagation of thermal runaway. The spread of the reaction zone is highlighted in semi-transparent red, and the yellow arrow indicates a major shift of active material toward the vent. Inset: Illustration showing the location of nail penetration. Real-time radiographs are provided as Supplementary Movie 1.

propagated in the longitudinal direction appeared to be dependent on radial position, where the layers closest to the cell casing exhibited the fastest propagation of thermal runaway. This may be a result of the highest temperatures being reached during the penetration of the outer layers, before the short circuit was softened by being spread across a greater area. By the time the inner layers were penetrated the increased shorting area had already significantly softened the shorting effect. This led to the formation of a conically-shaped reaction zone during the first $0.3 \mathrm{~s}$ of thermal runaway.

Thereafter, the fluidized, broken-down active materials flowed toward the vent of the cell (at $2.6545 \mathrm{~s}$ in Figure 3). This flow of material from the point of initiation toward the vent carried the heat of reaction in that direction, and is expected to have caused the surface temperature profile observed for Position 1 in Figure 2, where a high temperature region extended from the point of penetration to the cell-header. In Supplementary Movie 1, the visualization of fluidization of reactants and fluid dynamics thereafter offers valuable insight into the mechanisms of thermal runaway propagation and heat dissipation and may be of interest to the multi-physics modelling community.

Position 2. In Figure 4, penetration of the cell in the longitudinal direction through the base plate of the 18560 cell is shown. Using hydraulic clamps, this cell was suspended ca. $1 \mathrm{~cm}$ above the bottom surface of the containment vessel so that the cell venting process was not hindered. The tensile strain and consequent mechanical failure of the electrode assembly observed for Position 1 did not occur when the battery was punctured in the longitudinal direction. The nail inserted itself between the layers of the electrode assembly without any apparent crushing or tearing. The rate of propagation of thermal runaway in the longitudinal direction was not captured due to the tip of the nail rapidly leaving the FOV in Figure 4 and Supplementary Movie 3. However, the first significant signs of thermal runaway propagation in the radial direction occurred ca. $0.4 \mathrm{~s}$ after the nail penetrated through the base plate (at $1.9075 \mathrm{~s}$ in Figure 4).

When significant degradation of active material was observed, the base plate of the cell rose and domed outwards (see $1.9075 \mathrm{~s}$ in

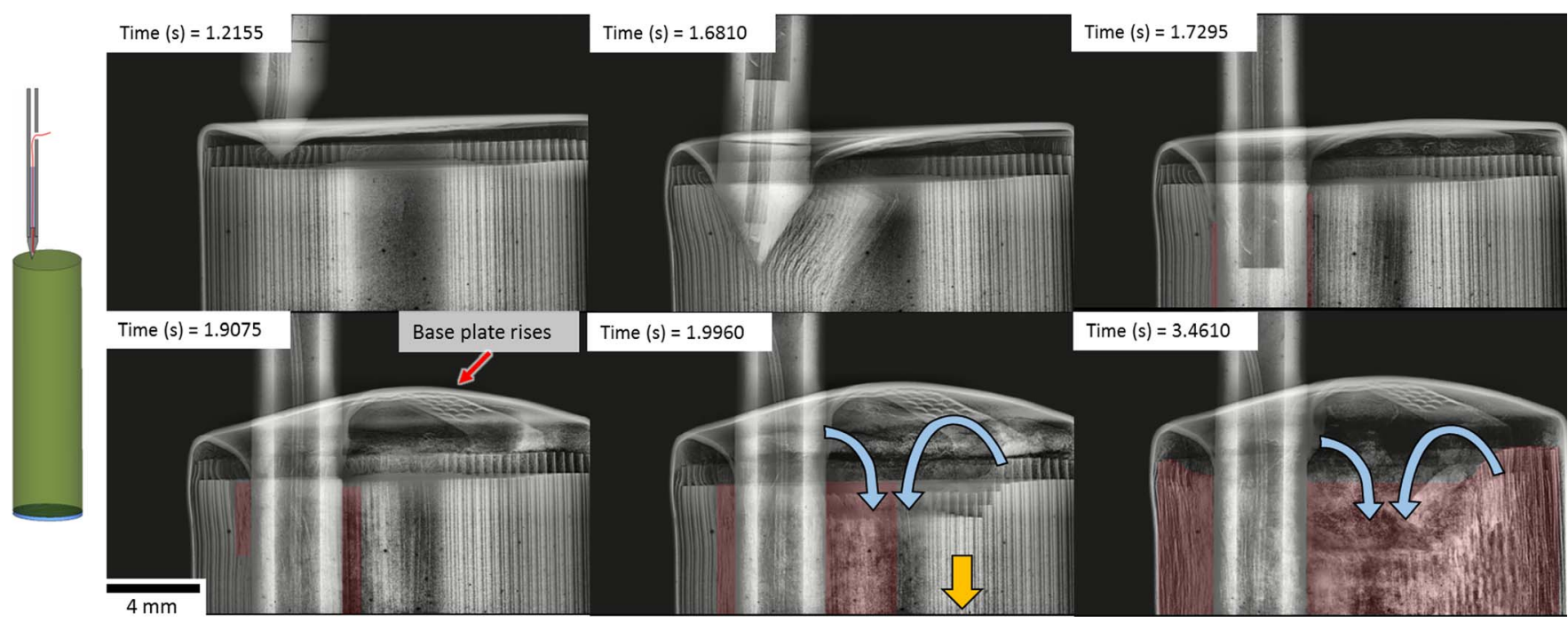

Figure 4. Time-stamped radiographs of nail penetration of an 18650 cell in Position 2 showing initiation and propagation of thermal runaway. The spread of the reaction zone is highlighted in semi-transparent red, the yellow arrow indicates a major shift of active material toward the vent, and the blue arrows represent the path of the broken-down fluidized material. Inset: Illustration showing the location of nail penetration. Real-time radiographs are provided as Supplementary Movie 3. 


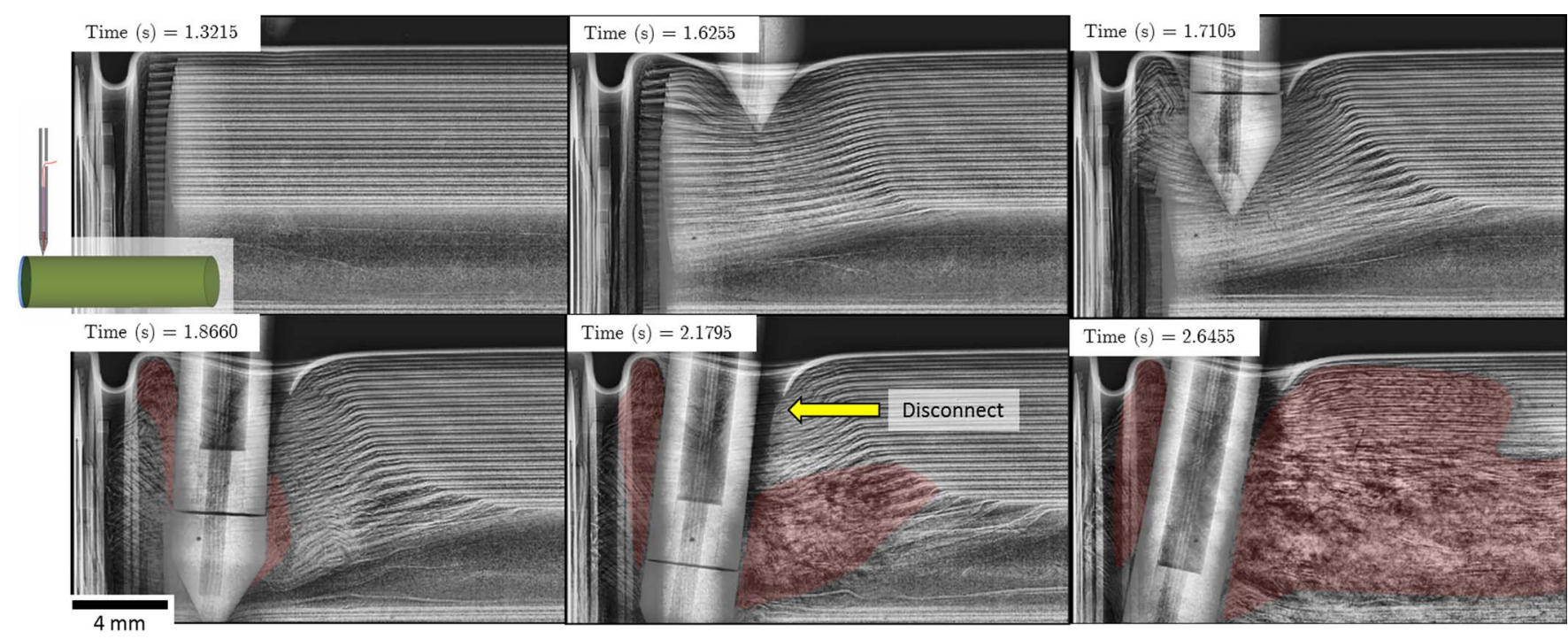

Figure 5. Time-stamped radiographs of nail penetration of an 18650 cell in Position 3 showing initiation and propagation of thermal runaway. The spread of the reaction zone is highlighted in semi-transparent red, and the yellow arrow highlights the region of the electrode assembly that loses contact with the nail. Inset: Illustration showing the location of nail penetration. Real-time radiographs are provided as Supplementary Movie 5.

Figure 4). This was caused by the buildup of pressure at the base of the cell, which caused a major shift of the inner layers of the electrode assembly toward the cell-header, as highlighted by the yellow arrow at $1.9960 \mathrm{~s}$ in Figure 4.

As well as causing the electrode assembly to shift, the pressure buildup at the base of the cell caused a high flow rate of gas through the core of the assembly, which led to peeling of the inner electrode layers as shown previously. ${ }^{8}$ The direction of gas flow is apparent in Supplementary Movie 3 where fragments of broken-down material detached from within the observable electrode assembly, were carried out of the spiral-wound layers toward the base of the cell, and thereafter, toward the vent of the cell through the vacant core, as illustrated by the blue arrows in Figure 4. This suggests that gases generated within the electrode assembly in the bottom portion of the cell initially flowed toward the vacant base of the casing and thereafter changed direction toward the vent, a tortuous path for pressure relief. This buildup of pressure at the base of the cell and consequent doming is not unique to mechanical abuse scenarios; similar shifts of the electrode assembly have also been observed under thermal abuse conditions $^{8,17}$ and are expected to be the primary cause of cell rupture and ejection of their contents.

The gas flow and evident pressure rise observed here highlights the necessity for a clear escape path of gases from the base of the cell to the vent, thereby avoiding extreme pressure buildup and explosion. This observation supports the case for the presence of an internal mandrel, which was previously shown to facilitate maintaining an opening for gas and fluidized material to escape. ${ }^{8}$ Gas generation and the consequent uneven and dynamic pressure distribution within cells during failure is an additional important consideration for multi-physics models of battery failure, particularly since it is local pressure differences that drive the flow of fluidized material, the venting process, as well as movement of the bulk electrode assembly.

Position 3. For this position, the cell was oriented horizontally (Figure 5) and the point of penetration was near the top of the electrode assembly which, in contrast to penetration mid-way along the cell (Position 1), generated an asymmetric counteracting force exerted by the assembly on the nail. This was due to the electrode assembly being relatively free to move near the cell-header but with little freedom to displace toward the base of the cell, resulting in greater compression of the electrode material on the inner side of the nail as it passed through. As a consequence the nail was deflected toward the less compressed side, and the asymmetric strain resulted in offset crack propagation from the tip of the nail. This is in contrast to the perpendicular crack propagation observed during penetration in Position 1 (Figure 3). The tip of the nail was deflected toward the vacant region between the top of the electrode assembly and the cell-header, departing from the short-circuiting reaction zone. Hence, the anomalous convergence of internal and surface temperatures in Figure 2a can be explained by the thermocouple coming to rest in a cooler, non-reacting region, with relatively efficient heat dissipation through the cell-header.

The temperature profiles shown in Figure 2 also highlight that, following penetration at Position 3 , the cell incurred the least extreme temperature rise with a maximum surface temperature of only ca. $500^{\circ} \mathrm{C}$. Longitudinally, thermal runaway mostly propagated in one direction only; toward the base of the cell. This was due to the nail penetrating the top of the assembly and hence there being little material through which thermal runaway could propagate toward the cellheader. The overall rate of heat production during thermal runaway would be expected to be significantly reduced due to less material exothermically breaking down at any point in time. The breakdown and ejection of active materials continued for ca. $1 \mathrm{~s}$ when penetrated at Position 1 (Supplementary Movie 1), whereas for Position 3 , breakdown and ejection continued for ca. $2.5 \mathrm{~s}$ (Supplementary Movie 5). Hence, assuming equal rates of propagation in both longitudinal directions, the position that resulted in the highest rate of heat generation, from beginning to end of the thermal runaway process, was mid-way along the electrode assembly (Position 1). The ejection process and heat dissipation during penetration at Position 3 is expected to be most effective due to the reaction zone being closest to the vent, allowing unhindered escape of the fluidized broken-down material. This is in contrast to penetration at the base of the cell (Position 2), where broken-down material followed a more tortuous path, first toward the base of the cell, and thereafter toward the cellheader via the vacant core of the electrode assembly.

The propagation of thermal runaway did not follow the conicalshaped reaction zone observed in Position 1 (Figure 3), which is thought to be due to the nail losing contact with the outer layers of the electrode assembly as it was deflected toward the cell-header (highlighted by an arrow at $2.1795 \mathrm{~s}$ in Figure 5). When the nail separated from the outer layers of the electrode assembly, the electrical contact for hard short-circuiting to occur was lost and consequently thermal runaway was mitigated at that location. The nail maintained contact with the inner layers, from where thermal runaway propagated uninterrupted. However, in the repeat test (Supplementary Movie 6) the nail penetrated at a slightly lower position toward the base of the cell and did not lose contact with the outer layers of the electrode 


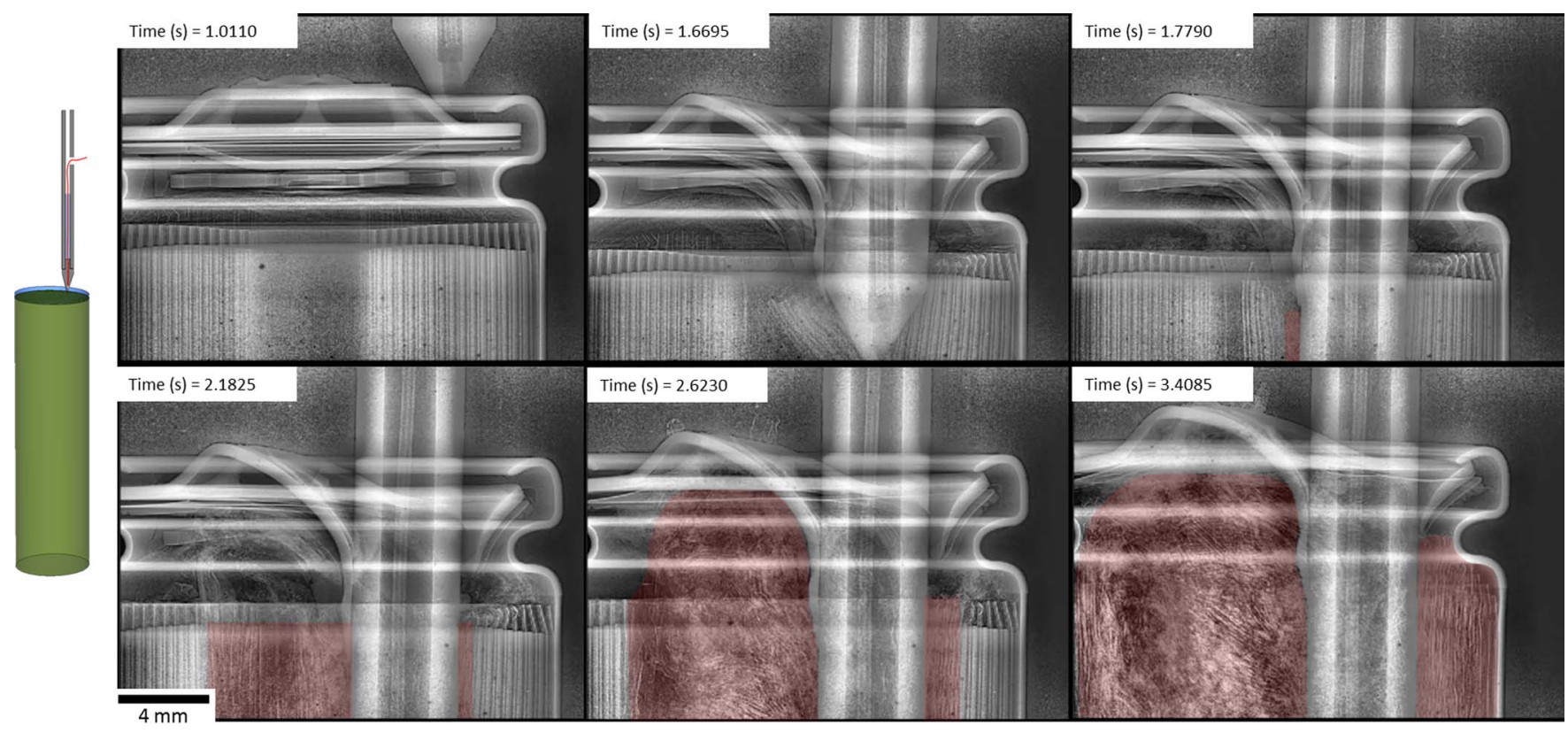

Figure 6. Time-stamped radiographs of nail penetration of an 18650 cell in Position 4 showing initiation and propagation of thermal runaway. The spread of the reaction zone is highlighted in semi-transparent red, and the yellow arrow indicates a major shift of active material toward the vent. Inset: Illustration showing the location of nail penetration. Real-time radiographs are provided as Supplementary Movie 7.

assembly, and hence the expected conical-shaped reaction zone was observed.

For this particular cell, the electrode layers were able to collapse into the vacant core. It is expected that the presence of an internal mandrel would significantly affect the mechanism of thermal runaway propagation; the electrode assembly layers would be pinched between the mandrel and the nail, leading to additional shorting as a result of compressive damage. ${ }^{7}$

Position 4. Here, the 18650 cell was vertically oriented and the nail penetrated through the cell-header (Figure 6). Penetration tests where the axis of penetration is parallel to the longitudinal direction are expected to result in a relatively hard short circuit due to fewer layers making contact. Contrary to the expectation that longitudinal penetration leads to a more severe thermal runaway process, it was observed that the rate at which thermal runaway spreads throughout the cell appears to be greater when the nail penetrates in the radial direction (Positions 1 and 3). Since propagation of thermal runaway occurs at a significantly slower rate in the radial direction than the longitudinal and azimuthal directions, ${ }^{8,23,24}$ the rate of propagation is more dependent on the number of layers in which thermal runaway is initiated along the radial direction. This indicates that, for this particular cell, propagation of thermal runaway was limited by the rate of propagation radially, rather than the temperature reached in the vicinity of the short.

The temperature at the short-circuiting location was the highest for this penetration where the cell was placed in the vertical position, reaching $920^{\circ} \mathrm{C}$ (Figure 2). The higher temperature did not appear to have significantly accelerated propagation in the radial direction. Despite the temperature being lower across the shorting area when penetrated in the vertical direction, a sufficient temperature for thermal runaway to initiate was still reached across multiple layers in the radial direction. Thereafter, the greater rate of propagation longitudinally and azimuthally, arising from each layer shorted along the radial direction, resulted in a relatively fast propagation of thermal runaway throughout the cell.

Similar to Position 3, the initiation and propagation of thermal runaway next to the vent allowed a relatively rapid escape of brokendown fluidized material, and hence improved heat dissipation into the environment (Figure 6). This is in contrast to the buildup of pressure and displacement of the intact electrode assembly when penetrated through the base of the cell (Position 2). Avoiding the shift of the intact electrode assembly in this case would have helped avoid the risk of the vent becoming clogged by the intact assembly and subsequent rupture of the cell. ${ }^{8}$ From Supplementary Movie 7, the cell still showed evidence of reactions occurring $2 \mathrm{~s}$ after thermal runaway first initiated, indicating a slower rate of completion of the thermal runaway process compared to penetration at Position 1.

Nail penetration tests: a discussion on variability and representativeness. - This work demonstrates the degree of variability that can occur during nail penetration tests and highlights that consistent reproducible behavior can be difficult to achieve. The wide area over which the short circuit spreads during nail penetration tests, as well as the nail itself acting as a local heat sink, counteracts the extreme temperatures that would otherwise be reached during internal short circuits, where a much smaller area between a single positive and negative electrode layer makes unintentional contact. By reducing the local temperature, the cell becomes less likely to undergo side-wall rupture, which is one of the greatest safety concerns for battery pack manufacturers due to the increased risk of cell-to-cell propagation of thermal runaway. The penetrating nail also creates an alternative escape path through which generated gases can flow, interfering with the pressure drop toward the vent, and potentially reducing the pressure buildup that the vent would otherwise experience.

The nail penetration test inherently imposes mechanical interference on the failure mechanism of the cell, compared to that induced by a local short circuit. This work has shown how the location at which the nail penetrates, as well as the orientation of the cell, can influence the mechanism of failure. When the cell is penetrated while placed in the horizontal position (Positions 1 and 3) the nail pins the electrode assembly and obstructs the ejection of cell contents by providing an obstruction perpendicular to the direction of flow. This prevents the safety devices at the cell header from handling what is otherwise expected to be a more severe ejection process. By resisting movement of the electrode assembly toward the vent of the cell, the nail penetration test could lead to erroneous characterization of the tendency of a particular cell design to undergo vent-clogging, bursting, and generation of projectiles. The position at which the cell is penetrated while placed horizontally also influences the rate of thermal runaway propagation; penetration at either end of the electrode assembly induces 
thermal runaway predominantly in the direction toward the opposite end of the cell, whereas penetration mid-way along the assembly allows more prolonged propagation in both directions. When the cell is penetrated while placed in the vertical position (Positions 2 and 4), the nail is parallel to the electrode assembly and to the direction of flow. In the vertical position, the electrode assembly is not pinned and can shift toward the vent more freely (as seen in Position 2). However, in the vertical position, the nail may introduce greater shear force and compression of the electrode assembly against the wall of the cell. Penetrating through the base of the cell presents greater scope for testing the efficacy of safety devices in the header of the cell, as well as testing the tendency for vent clogging and cell rupture.

This work clearly demonstrates the variability associated with the nail penetration test and its sensitivity to penetration location and cell orientation. Internal cylindrical mandrels ${ }^{8,17}$ occupying the core of the electrode assembly may further affect this variation by introducing the possibility of the electrode assembly being pinched between the nail and the rigid mandrel. At present, nail penetration tests are frequently used to assess the safety of commercial cells and module designs with regard to thermal runaway. As shown here, the combination of the nail softening the short circuit, introducing a heat sink to the region of initiation, limiting the movement of material within the cell, and relieving pressure buildup makes nail penetration an ineffective means of simulation of worst-case failure scenarios. Due to the variation in failure mechanisms and the reduced severity of failure, utilising nail penetration for the purpose of safety tests raises the possibility of erroneous determination of cell or module safety, since vulnerabilities to extreme circumstances such as side-wall rupture or bursting may still exist.

\section{Conclusions}

Internal temperatures were found to be highest for nail penetrations where the cell was penetrated while placed in a vertical position. However, despite the higher temperatures being reached locally at the shorting region, the rate of propagation throughout the cell was relatively slow when compared to cells penetrated while placed horizontally. The propagation of thermal runaway throughout the cell appeared to be limited by the slow heat dissipation and spread of thermal runaway reactions in the radial direction, rather than the temperature reached at the point of initiation. The fact that higher temperatures were reached locally during vertical (longitudinal) penetrations may have implications for the risk of thermal runaway at lower states of charge, where initiation may be limited by local temperatures - that is to say, for low state of charge cells, a vertical penetration might be more likely to result in thermal runaway than a horizontal one.

The high variability of internal structural dynamics that was observed between the four penetration positions directly influenced the failure mechanisms during thermal runaway. Penetration through the base of the cell and initiation of thermal runaway at the bottom of the electrode assembly resulted in a local pressure buildup and a rapid flow of material through the vacant core of the assembly. This caused the bulk electrode assembly to shift toward and clog the vent, which is a known cause of cells bursting. The varying rate of propagation and ejection of broken-down material also influenced the external temperature profiles that were non-uniform and showed that the hottest regions followed the path of ejection from the point of penetration.
This has implications for temperature measurement strategies on the surface of the cell where temperatures can vary by $>100^{\circ} \mathrm{C}$.

A discussion on the representativeness of nail penetration tests of in-field failure scenarios highlights the potential for erroneous determination of risks posed by cells undergoing thermal runaway and the need for a method to induce internal short circuits without an intrusive object interfering with the failure mechanism itself. This would allow in-field failures to be more accurately characterized and the efficacy of safety devices against worst-case failure scenarios to be examined.

\section{Acknowledgments}

The authors acknowledge funding from the EPSRC (EP/N032888/1, EP/M009394/1), the Royal Academy of Engineering, the STFC (ST/N002385/1) and the National Measurement System. These experiments were performed at ID19 at the ESRF (Grenoble, France).

\section{References}

1. R. Spotnitz and J. Franklin, Journal of Power Sources, 113, 81 (2003).

2. Y. Chen and J. W. Evans, Journal of The Electrochemical Society, 143, 2708 (1996)

3. C. -H. Doh, D. -H. Kim, H. -S. Kim, H. -M. Shin, Y. -D. Jeong, S. -I. Moon, B. -S. Jin, S. W. Eom, H. -S. Kim, K. -W. Kim, D. -H. Oh, and A. Veluchamy, Journal of Power Sources, 175, 881 (2008)

4. A. W. Golubkov, D. Fuchs, J. Wagner, H. Wiltsche, C. Stangl, G. Fauler, G. Voitic, A. Thaler, and V. Hacker, RSC Advances, 4, 3633 (2014).

5. A. W. Golubkov, S. Scheikl, R. Planteu, G. Voitic, H. Wiltsche, C. Stangl, G. Fauler, A. Thaler, and V. Hacker, RSC Advances, 5, 57171 (2015)

6. C. F. Lopez, J. A. Jeevarajan, and P. P. Mukherjee, Journal of The Electrochemical Society, 162, A1905 (2015)

7. J. Lamb and C. J. Orendorff, Journal of Power Sources, 247, 189 (2014).

8. D. P. Finegan, E. Darcy, M. Keyser, B. Tjaden, T. M. M. Heenan, R. Jervis, J. J. Bailey, R. Malik, N. T. Vo, O. V. Magdysyuk, R. Atwood, M. Drakopoulos, M. DiMichiel, A. Rack, G. Hinds, D. J. L. Brett, and P. R. Shearing, Energy \& Environmental Science, 10, 1377 (2017)

9. C. J. Orendorff, E. P. Roth, and G. Nagasubramanian, Journal of Power Sources, 196, 6554 (2011).

10. T. Wierzbicki and E. Sahraei, Journal of Power Sources, 241, 467 (2013).

11. E. Sahraei, J. Campbell, and T. Wierzbicki, Journal of Power Sources, 220, 360 (2012).

12. E. Sahraei, J. Meier, and T. Wierzbicki, Journal of Power Sources, 247, 503 (2014).

13. C. Zhang, S. Santhanagopalan, M. A. Sprague, and A. A. Pesaran, Journal of Power Sources, 298, 309 (2015).

14. H. Maleki and J. N. Howard, Journal of Power Sources, 191, 568 (2009).

15. K. -C. Chiu, C. -H. Lin, S. -F. Yeh, Y. -H. Lin, and K. -C. Chen, Journal of Power Sources, 251, 254 (2014)

16. W. Zhao, G. Luo, and C. -Y. Wang, Journal of The Electrochemical Society, 162, A207 (2015).

17. D. P. Finegan, M. Scheel, J. B. Robinson, B. Tjaden, I. Hunt, T. J. Mason, J. Millichamp, M. Di Michiel, G. J. Offer, G. Hinds, D. J. L. Brett, and P. R. Shearing, Nat Communications, 6 (2015).

18. D. P. Finegan, M. Scheel, J. B. Robinson, B. Tjaden, M. Di Michiel, G. Hinds, D. J. L. Brett, and P. R. Shearing, Physical Chemistry Chemical Physics, 18, 30912 (2016).

19. T. D. Hatchard, S. Trussler, and J. R. Dahn, Journal of Power Sources, 247, 821 (2014)

20. P. Arora and Z. Zhang, Chemical Reviews, 104, 4419 (2004).

21. D. P. Finegan, S. J. Cooper, B. Tjaden, O. O. Taiwo, J. Gelb, G. Hinds, D. J. L. Brett, and P. R. Shearing, Journal of Power Sources, 333, 184 (2016)

22. J. Cannarella, X. Liu, C. Z. Leng, P. D. Sinko, G. Y. Gor, and C. B. Arnold, Journal of The Electrochemical Society, 161, F3117 (2014).

23. S. Santhanagopalan, P. Ramadass, and J. Zhang, Journal of Power Sources, 194, 550 (2009)

24. G. -H. Kim, A. Pesaran, and R. Spotnitz, Journal of Power Sources, 170, 476 (2007). 